\title{
Delay in initiation of postoperative adjuvant chemotherapy with S-1 monotherapy and prognosis for gastric cancer patients: analysis of a multi-institutional dataset
}

\author{
Koki Nakanishi ${ }^{1} \cdot$ Mitsuro Kanda $^{1}$ (D) Seiji Ito ${ }^{2} \cdot$ Yoshinari Mochizuki $^{3} \cdot$ Hitoshi Teramoto $^{4} \cdot$ Kiyoshi Ishigure $^{5}$. \\ Toshifumi Murai ${ }^{6} \cdot$ Takahiro Asada $^{7} \cdot$ Akiharu Ishiyama $^{8} \cdot$ Hidenobu Matsushita $^{9} \cdot$ Chie Tanaka $^{1}$. \\ Daisuke Kobayashi ${ }^{1} \cdot$ Michitaka Fujiwara $^{1} \cdot$ Kenta Murotani $^{10} \cdot$ Yasuhiro Kodera $^{1}$
}

Received: 30 January 2019 / Accepted: 30 March 2019 / Published online: 6 April 2019

(c) The International Gastric Cancer Association and The Japanese Gastric Cancer Association 2019

\begin{abstract}
Background This study aimed to evaluate whether the timing of initiating postoperative chemotherapy with S-1 monotherapy affects gastric cancer patients' prognosis.

Methods A multi-institution dataset identified patients with pStage II or III gastric cancer who received S-1 monotherapy for over 6 months after curative resection between 2010 and 2014. Patients were divided into three groups based on the timing of S-1 monotherapy initiation. Prognostic factors for relapse-free survival (RFS) were investigated.

Results We classified 401 patients into groups as follows: S- 1 administered within 6 weeks $(n=247)$, between 6 and 8 weeks $(n=95)$, and after 8 weeks $(n=59)$. The RFS times were not significantly different in the within 6 weeks group and the between 6 and 8 weeks group, but the after 8 weeks group had a shorter RFS time compared with the other two groups (within 6 weeks group vs. after 8 weeks group; $P=0.0044$ ). By disease stage, this trend was the same. The multivariable analysis showed that a larger tumor size $(\geq 50 \mathrm{~mm})$, pStage III, and the after 8 weeks group were independent prognostic factors for RFS (after 8 weeks group: hazard ratio, 2.05; $P=0.0069$ ). The prevalence of hematogenous metastasis as the initial recurrence site increased by delayed initiation of S-1. A forest plot revealed that delayed administration after 8 weeks was associated with a greater risk of recurrence in most subgroups.

Conclusions Postoperative chemotherapy with S-1 monotherapy for gastric cancer is recommended to begin within 8 weeks after surgery.
\end{abstract}

Keywords Gastric cancer $\cdot$ Timing of initiation $\cdot S-1 \cdot$ Prognosis $\cdot$ Postoperative chemotherapy

Electronic supplementary material The online version of this article (https://doi.org/10.1007/s10120-019-00961-9) contains supplementary material, which is available to authorized users.

Mitsuro Kanda

m-kanda@med.nagoya-u.ac.jp

1 Department of Gastroenterological Surgery (Surgery

II), Nagoya University Graduate School of Medicine, 65

Tsurumai-cho, Showa-ku, Nagoya 466-8550, Japan

2 Department of Gastroenterological Surgery, Aichi Cancer Center, Nagoya, Japan

3 Department of Surgery, Komaki Municipal Hospital, Komaki, Japan

4 Department of Surgery, Yokkaichi Municipal Hospital, Yokkaichi, Japan
5 Department of Surgery, Konan Kosei Hospital, Konan, Japan

6 Department of Surgery, Ichinomiya Municipal Hospital, Ichinomiya, Japan

7 Department of Surgery, Gifu Prefectural Tajimi Hospital, Tajimi, Japan

8 Department of Surgery, Okazaki City Hospital, Okazaki, Japan

9 Department of Surgery, Tosei General Hospital, Seto, Japan

10 Biostatistics Center, Graduate School of Medicine, Kurume University, Kurume, Japan 


\section{Introduction}

Gastric cancer is the fifth most common malignancy worldwide [1]. Surgical resection remains the most promising treatment for resectable gastric cancers, but surgical resection alone does not prevent gastric cancer recurrence. In East Asia, adjuvant chemotherapy, including S-1 (an oral fluoropyrimidine derivative) monotherapy for 1 year following curative resection, is the standard treatment for pathological stage II and III gastric cancer [2], and its survival benefit has been shown [3]. The aim of adjuvant chemotherapy after surgery is to eradicate residual microscopic cancer cells, and in theory, it must be started as soon as possible and continued intermittently [4-6].

There have been two issues regarding postoperative adjuvant chemotherapy with S-1 monotherapy: duration of administration and timing of initiation after surgery. The effect of these issues on prognosis has not been previously investigated. The duration of postoperative chemotherapy with S-1 monotherapy for gastric cancer patients has been recommended as 1 year, based on the ACTS-GC trial results [3]. Recently, a Japanese large-scale retrospective study reported that a duration of more than 6 months correlated with a good prognosis [7]. A prospective randomized controlled trial to confirm non-inferiority of relapse-free survival (RFS) for S-1 administered for 6 months-1 year in pathological stage II gastric cancer patients was conducted [8], but the trial failed. Thus, the ideal duration remains 1 year. Proper timing of postoperative chemotherapy initiation with S-1 monotherapy has not been fully evaluated. Based on the rules of the ACTSGC trial [3], it is recommended by the Japanese gastric cancer treatment guidelines to start within 6 weeks after surgery, but no persuasive evidence was quoted [2]. There were a few retrospective studies that suggest a correlation between the timing of initiation and prognosis, while some studies reported that delayed initiation was associated with an unfavorable prognosis $[9,10]$, and other studies reported that the timing of initiation was not correlated with prognosis [7]. This correlation remains controversial. Moreover, in clinical practice, not all patients can start S-1 monotherapy within 6 weeks because of postoperative complications and poor general condition that requires postoperative recovery until $\mathrm{S}-1$ administration is available. Additionally, a poor prognosis could be a result of postoperative complications and poor general condition [11-13], and whether delayed initiation alone can affect the outcome is unknown. This issue has never been addressed by prospective randomized controlled trials, and a single-institution study had a small number of patients.

To address these issues, we compiled a multicenter dataset comprising a larger number of patients with stage
II and III gastric cancer who underwent gastrectomy and investigated whether the timing of postoperative chemotherapy initiation with S-1 monotherapy affects gastric cancer patient prognosis.

\section{Patients and methods}

\section{Patient selection}

We retrospectively reviewed the clinical data from 3484 patients who underwent gastrectomy for gastric cancer between January 2010 and December 2014, as acquired from medical records at nine institutions. We retrieved eligible patients for analysis based on the following criteria: no preoperative treatment, pathologically proven gastric adenocarcinoma, R0 gastrectomy with proper systematic lymphadenectomy performed according to the Japanese Gastric Cancer Treatment Guidelines [2], pathological stage II/III except T1 and T3N0 gastric cancer based on the 8th edition of TNM staging system of the Union for International Cancer Control [14], received postoperative chemotherapy with S-1 monotherapy, and sufficient data for analysis. Patients who underwent extended surgery (pancreaticoduodenectomy) or limited surgery without systemic lymphadenectomy were excluded from the study. It is already shown that an administration period of more than 6 months is a significant prognostic factor [7, 8], and an administration period of less than 6 months does not allow sufficient benefit from S-1. Thus, we excluded the patients who withdrew from S-1 monotherapy within 6 months for reasons other than recurrence in this study.

\section{Ethics}

This study conformed to the World Medical Association Declaration of Helsinki-Ethical Principles for Medical Research Involving Human Subjects ethical guidelines. Written informed consent for surgery and use of clinical data was obtained from patients as required by the Institutional Review Board of each participating institution. We applied an opt-out recruitment strategy in accordance with the Japanese government's policy because we conducted this clinical research using the only retrospective clinical data without intervention. The purpose, design, and objectives of the study were posted on our homepage (https://www.med.nagoya-u.ac.jp/medical_J/ethics/rinsy oukansatsu.html.) to provide an opportunity for patients to decline to participate in our study. 


\section{Patient management}

The postoperative complications were evaluated in accordance with the Clavien-Dindo classification $[15,16]$. The patients' disease stages were determined in accordance with the 8th edition of the TNM staging system of the Union for International Cancer Control [14]. Histological type was classified based on the Japanese Gastric Cancer Association classification system [17], in which tubular and papillary adenocarcinoma are defined as differentiated adenocarcinomas, while poorly differentiated adenocarcinoma, signet-ring cell carcinoma, and mucinous adenocarcinoma are defined as undifferentiated adenocarcinomas. Postoperative chemotherapy with S-1 monotherapy has been recommended to all patients with stage II/III except T1 and T3N0 gastric cancer, unless contraindicated by the patient's condition [18]. S-1 was administered based on body surface area as follows: $<1.25 \mathrm{~m}^{2}$ (80 mg daily), $1.25-1.50 \mathrm{~m}^{2}(100 \mathrm{mg}$ daily), and $\geq 1.50 \mathrm{~m}^{2}$ (120 mg daily) [3]. The S-1 monotherapy regimen was administered for 4 weeks followed by a rest for 2 weeks or 2 weeks followed by a rest for 1 week. This cycle was repeated primarily for 1 year after surgery. Toxicity of chemotherapy was assessed based on the Common Toxicity Criteria of the National Cancer Institute, version 4.0. If patients had grade 3 or 4 (greatest possible grade) hematologic toxic effects or grade 2, 3, or 4 nonhematologic toxic effects, their daily dosage was reduced, or their treatment was postponed or stopped based on each physician's judgment [18]. During S-1 administration, patients underwent hematologic tests and assessments of clinical symptoms each cycle. After S-1 administration, patient follow-up was performed in accordance with the Japanese gastric cancer treatment guidelines [2], as follows: physical examinations and laboratory tests were performed every 3 months for the first 3 years and then every 6 months until 5 years after surgery, enhanced CT (chest and abdominal cavity) was conducted every 6 months for 5 years, and endoscopy was performed 1,3 , and 5 years after the non-total gastrectomy. Patients were diagnosed with a relapse when signs or symptoms indicated a possible recurrence.

\section{Patient groups}

Patients were classified into three groups based on the interval between the surgery and initiation of postoperative chemotherapy with S-1 monotherapy as follows: administration within 6 weeks, administration between 6 and 8 weeks, and administration after 8 weeks. The cutoff time of 6 weeks was set based on the Japanese gastric cancer treatment guidelines [2] and the cutoff time of 8 weeks was set based on the timing for colon cancer [19], which are both gastrointestinal tract cancers.

\section{Statistical analysis}

Correlations between each group and clinicopathological variables were analyzed by the $\chi^{2}$ and Fisher's exact tests for categorical variables and the Mann-Whitney $U$ test for continuous variables. Overall survival (OS) and relapse-free survival (RFS) were calculated using the Kaplan-Meier method and differences in survival curves were analyzed using the log rank test and the Bonferroni adjustment for multiple comparisons. A multivariate analysis was performed using the Cox proportional hazard model and the logistic regression model, after relevant prognostic variables had been defined by the univariate analysis. The significance of the covariates was tested using the Wald test. Data were analyzed using JMP ver13 software (JMP, SAS Institute, Cary, North Carolina, USA). A $P$ value of $<0.05$ was considered to indicate statistically significant differences.

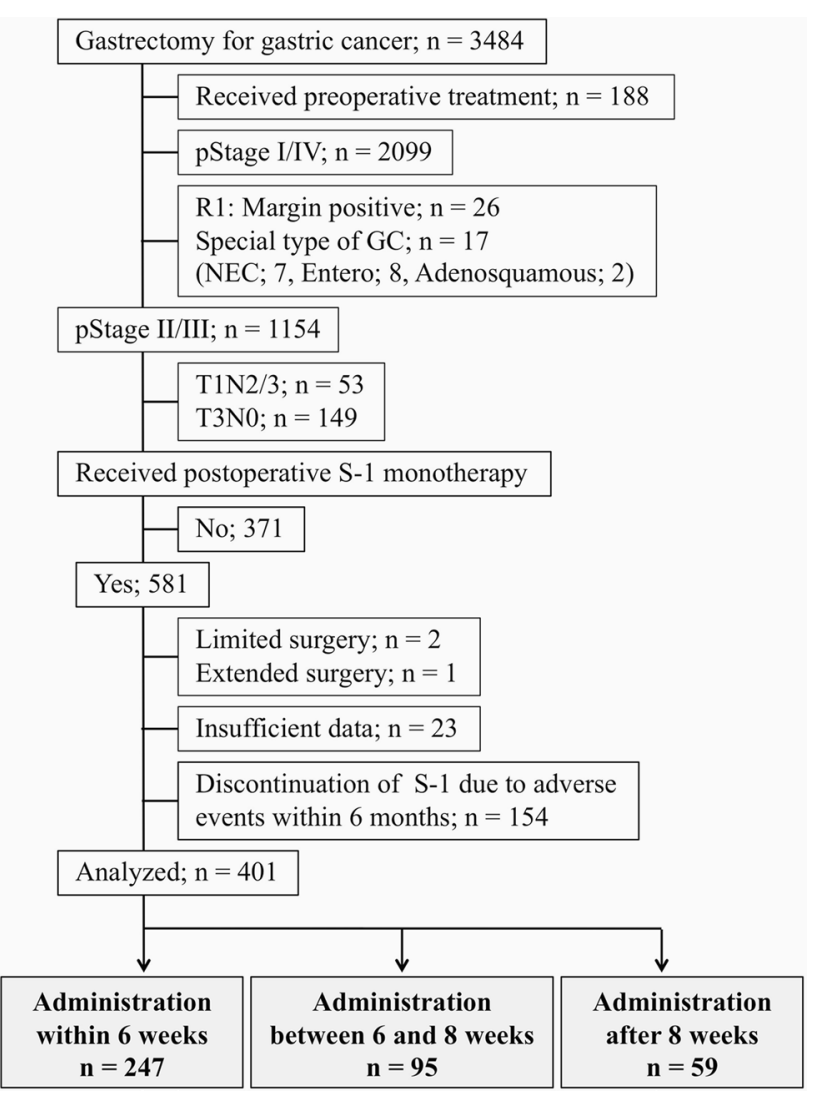

Fig. 1 Flow-chart of patient enrollment. GC gastric cancer, NEC neuroendocrine carcinoma, Entero carcinoma with enteroblastic differentiation, Adenosquamous Adenosquamous carcinoma 


\section{Results}

\section{Patients' characteristics}

The patients' flow chart is shown in Fig. 1. Four hundred and one patients were divided into three groups as follows: 247 patients with S-1 administration within 6 weeks, 95 patients with S-1 administration between 6 and 8 weeks, and 59 patients with S-1 administration after 8 weeks. The average time to $\mathrm{S}-1$ administration for each group was $5.1 \pm 0.9,7.4 \pm 0.5$, and $11.9 \pm 3.3$ weeks, respectively. The overall patient clinical and histopathological characteristics are summarized in Table 1 by group. Overall, 138 (34.4\%) had pStage II disease and 263 (65.6\%) had pStage III disease. There were no differences in sex distribution, Eastern Cooperative Oncology Group Performance Status (ECOG-PS) score, surgical approach, tumor size, macroscopic type, $\mathrm{pT}$-stage, $\mathrm{pN}$-stage, or disease stage. Although not significantly different, total gastrectomy tended to increase the administration between 6 and 8 weeks and after 8 weeks. Patients with S-1 administration after 8 weeks had a significantly greater proportion of elderly patients, differentiated type of tumor histology, higher incidence of postoperative complications, and longer hospital stay after surgery. The prevalence of grade III postoperative complications was higher in the administration after 8 weeks group compared with the other groups [18 (38\%) patients with grade IIIa, 4 (7\%) patients with grade IIIb]. There was no difference in the postoperative complications rate using the surgical approach (open, $29 \%$; laparoscopic, $25 \% ; P=0.6449$ ). The median followup period for patients in this study was 45.5 months (range 3.8-94 months).

\section{Distribution of S-1 monotherapy}

The distribution of the time to initiating $\mathrm{S}-1$ is shown in Fig. 2a. The median interval between surgery and S-1 initiation was 6 weeks. Nine patients started S-1 administration more than 16 weeks after surgery. There were 310 patients $(77.3 \%)$ who completed S-1 monotherapy for 1 year. S-1 monotherapy was discontinued because a relapse was detected, the physician's decision to discontinue treatment because of adverse events, the patient's decision, and other factors in 53, 28, 6, and 4 patients, respectively. Among the 53 patients who discontinued S-1 monotherapy because of recurrence, 19 patients were in the administration within 6 weeks, 17 patients were in the administration between 6 and 8 weeks, and 17 patients were in the administration after 8 weeks groups. The S- 1 monotherapy completion rate was $82.2 \%$ in the administration within 6 weeks, $76.8 \%$ in the administration between 6 and 8 weeks, and $59.3 \%$ in the administration after 8 weeks groups, and the rate was significantly lower in the administration after 8 weeks group $(P=0.0006$; Fig. 2b).

\section{Relapse and survival outcomes}

Relapse occurred in 136 patients (33.9\%). The RFS times for the administration within 6 weeks group and the administration between 6 and 8 weeks group were not significantly different, but that of the administration after 8 weeks group was shorter compared with the other two groups (Fig. 3a: within 6 weeks vs. between 6 and 8 weeks, $P=0.7115$; within 6 weeks vs. after 8 weeks, $P=0.0044$; between 6 and 8 weeks vs. after 8 weeks, $P=0.3056$, respectively). The 5 -year RFS rates overall for all 401 patients were $67.2 \%$ in the administration within 6 weeks, $63.4 \%$ in the administration between 6 and 8 weeks, and $50.2 \%$ in the administration after 8 weeks groups. By disease stage, this trend was the same, and there was a significant difference between the administration within 6 weeks and the administration after 8 weeks groups for pStage III, but no significant difference for pStage II (Fig. 3b, pStage II: within 6 weeks vs. after 8 weeks, $P=0.1059$; Fig. 3c, pStage III: within 6 weeks vs. after 8 weeks, $P=0.0027$ ). The 5-year RFS rates for patients with stage II disease were $89.4 \%$ in the administration within 6 weeks, $89.0 \%$ in the administration between 6 and 8 weeks, and $75.0 \%$ in the administration after 8 weeks groups. The 5 -year RFS rates for the patients with stage III disease were $55.8 \%$ in the administration within 6 weeks, $52.5 \%$ in the administration between 6 and 8 weeks, and $32.8 \%$ in the administration after 8 weeks groups.

Disease-related death occurred in 94 patients (24.4\%). The disease-specific survival (DSS) in the administration within 6 weeks and the administration between 6 and 8 weeks groups were not significantly different, but that of the administration after 8 weeks was significantly shorter compared with the other two groups (Supplemental Fig. 1a, within 6 weeks vs. between 6 and 8 weeks, $P=0.4871$; within 6 weeks vs. after 8 weeks, $P=0.0003$; between 6 and 8 weeks vs. after 8 weeks, $P=0.1087$ ). By disease stage, this trend was the same, and there was a significant difference between the administration within 6 weeks and the administration after 8 weeks groups for pStage II and pStage III (Supplemental Fig. 1b, pStage II: within 6 weeks vs. after 8 weeks, $P=0.0225$; Supplemental Fig. 1c, pStage III: within 6 weeks vs. after 8 weeks, $P=0.0002$ ). The 5 -year DSS rates for patients with stage II disease were $96.3 \%$ in the administration within 6 weeks, $96.3 \%$ in the administration between 6 and 8 weeks, and $83.3 \%$ in the administration after 8 weeks groups. The 5 -year DSS rates for patients with stage III disease were $69.1 \%$ in the administration within 
Table 1 Patient characteristics categorized by time to initiation of S-1 monotherapy after surgery

\begin{tabular}{|c|c|c|c|c|}
\hline & $\begin{array}{l}\text { Within } 6 \text { weeks } \\
n=247\end{array}$ & $\begin{array}{l}\text { Between } 6 \text { and } \\
8 \text { weeks } \\
n=95\end{array}$ & $\begin{array}{l}\text { After } 8 \text { weeks } \\
n=59\end{array}$ & $P$ value \\
\hline Sex, $n(\%)$ & & & & 0.7776 \\
\hline Male & $174(70)$ & $66(69)$ & $44(75)$ & \\
\hline Female & $73(30)$ & $29(31)$ & $15(25)$ & \\
\hline Age, years & & & & 0.0104 \\
\hline Mean \pm SD & $65 \pm 10$ & $66 \pm 9$ & $69 \pm 8$ & \\
\hline \multicolumn{5}{|l|}{ Comorbidities, $n(\%)$} \\
\hline Diabetes mellitus & $34(14)$ & $17(18)$ & $13(22)$ & 0.2497 \\
\hline Cerebrovascular disease & $16(7)$ & $3(3)$ & $3(5)$ & 0.4772 \\
\hline Cardiopulmonary disease & $33(13)$ & $9(9)$ & $9(15)$ & 0.5131 \\
\hline ECOG-PS, $n(\%)$ & & & & 0.9181 \\
\hline $0-1$ & $240(97)$ & $92(97)$ & $57(97)$ & \\
\hline $2-3$ & $7(3)$ & $3(3)$ & $2(3)$ & \\
\hline Type of gastrectomy, $n(\%)$ & & & & 0.1762 \\
\hline Total gastrectomy & $91(37)$ & $41(43)$ & $29(49)$ & \\
\hline Non-total gastrectomy & $156(63)$ & $54(57)$ & $30(51)$ & \\
\hline Approach, $n(\%)$ & & & & 0.7306 \\
\hline Open & $228(92)$ & $90(95)$ & $55(93)$ & \\
\hline Laparoscopic & $19(8)$ & $5(5)$ & $4(7)$ & \\
\hline Dissected lymph node & & & & 0.9818 \\
\hline Mean \pm SD & $39.5 \pm 15.4$ & $39.3 \pm 15.6$ & $40.1 \pm 19.3$ & \\
\hline Tumor size, $\mathrm{cm}$ & & & & 0.6287 \\
\hline Mean \pm SD & $5.2 \pm 2.4$ & $5.6 \pm 3.0$ & $5.8 \pm 3.2$ & \\
\hline Macroscopic type, $n(\%)$ & & & & 0.4967 \\
\hline Bormann type $4 / 5$ & $16(6)$ & $9(9)$ & $6(10)$ & \\
\hline Others & $231(94)$ & $86(91)$ & $53(90)$ & \\
\hline Histologic type, $n(\%)$ & & & & 0.0102 \\
\hline Differentiated & $95(38)$ & $36(38)$ & $35(59)$ & \\
\hline Undifferentiated & $152(62)$ & $59(62)$ & $24(41)$ & \\
\hline Vessel infiltration, $n(\%)$ & & & & 0.2903 \\
\hline Present & $170(69)$ & $73(77)$ & $44(75)$ & \\
\hline Absent & $77(31)$ & $22(23)$ & $15(25)$ & \\
\hline Lymphatic infiltration, $n(\%)$ & & & & 0.1589 \\
\hline Present & $223(90)$ & $83(87)$ & $57(97)$ & \\
\hline Absent & $24(10)$ & $12(13)$ & $2(3)$ & \\
\hline pT stage, $n(\%)$ & & & & 0.5374 \\
\hline $\mathrm{T} 2$ & $43(17)$ & $14(15)$ & $8(14)$ & \\
\hline $\mathrm{T} 3$ & $71(29)$ & $35(37)$ & $22(37)$ & \\
\hline $\mathrm{T} 4$ & $133(54)$ & $46(48)$ & $29(49)$ & \\
\hline pN stage, $n(\%)$ & & & & 0.9015 \\
\hline N0 & $29(12)$ & $9(10)$ & $8(14)$ & \\
\hline N1 & $72(29)$ & $27(28)$ & $21(36)$ & \\
\hline $\mathrm{N} 2$ & $71(29)$ & $28(29)$ & $14(24)$ & \\
\hline N3 & $75(30)$ & $31(33)$ & $16(27)$ & \\
\hline pStage (UICC 8th), $n(\%)$ & & & & 0.6841 \\
\hline IIA & $26(11)$ & $7(7)$ & $5(8)$ & \\
\hline IIB & $60(24)$ & $21(22)$ & $19(32)$ & \\
\hline IIIA & $85(34)$ & $36(38)$ & $20(34)$ & \\
\hline IIIB & $55(22)$ & 18 (19) & $11(19)$ & \\
\hline IIIC & $21(9)$ & $13(14)$ & $4(7)$ & \\
\hline
\end{tabular}


Table 1 (continued)

\begin{tabular}{|c|c|c|c|c|}
\hline & $\begin{array}{l}\text { Within } 6 \text { weeks } \\
n=247\end{array}$ & $\begin{array}{l}\text { Between } 6 \text { and } \\
8 \text { weeks } \\
n=95\end{array}$ & $\begin{array}{l}\text { After } 8 \text { weeks } \\
n=59\end{array}$ & $P$ value \\
\hline \multicolumn{5}{|l|}{ Postoperative complications ${ }^{\mathrm{a}}(\%)$} \\
\hline II & $22(9)$ & $18(19)$ & $10(17)$ & \\
\hline IIIa & $6(2)$ & $16(17)$ & $18(31)$ & \\
\hline IIIb & $2(1)$ & $1(1)$ & $4(7)$ & \\
\hline IV & $0(0)$ & $1(1)$ & $2(3)$ & \\
\hline Total, $n(\%)$ & $30(12)$ & $36(38)$ & $34(58)$ & $<0.0001$ \\
\hline \multicolumn{5}{|l|}{ Type of complication (\%) } \\
\hline Anastomotic leakage & $1(0.4)$ & $7(7)$ & $3(5)$ & \\
\hline Intra-abdominal infection & $19(8)$ & $29(31)$ & $24(41)$ & \\
\hline Re-operation & $2(1)$ & $3(3)$ & $6(10)$ & \\
\hline Hospital stay after surgery, days & & & & $<0.0001$ \\
\hline Mean \pm SD & $13.2 \pm 4.2$ & $18.9 \pm 8.9$ & $26.0 \pm 17.7$ & \\
\hline
\end{tabular}

$S D$ standard deviation, ECOG-PS Eastern Cooperative Oncology Group Performance Status, UICC Union for International Cancer Control

${ }^{a}$ According to the Clavien-Dindo Classification
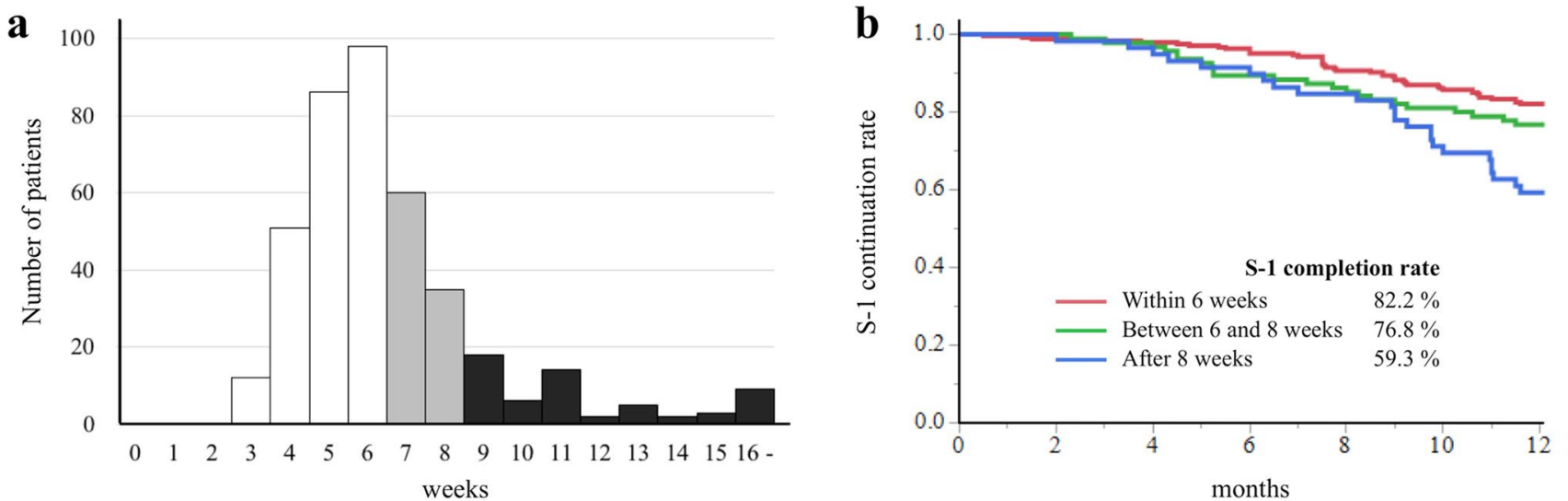

Fig. 2 a Interval time to initiation of S-1 monotherapy after surgery. This graph shows the distribution of S-1 monotherapy timing for all patients. b S-1 continuation rates for each group

6 weeks, $61.8 \%$ in the administration between 6 and 8 weeks, and $32.6 \%$ in the administration after 8 weeks groups.

\section{Univariate and multivariate analysis for relapse}

A univariate analysis identified the following significant predictive factors for RFS: larger tumor size $(\geq 5.0 \mathrm{~cm})$, vessel infiltration, lymphatic infiltration, pStage III, gastrectomy type (total gastrectomy), postoperative complications, and the administration after 8 weeks group. A multivariate analysis identified the following as significant factors: larger tumor size $[\geq 5.0 \mathrm{~cm}$ : hazard ratio (HR) $1.45 ; 95 \%$ confidence interval (CI) 1.01-2.10, $P=0.0458]$, pStage III (HR 3.85; 95\% CI 2.33-6.77, $P<0.0001$ ), and the administration after 8 weeks group (HR 2.05; 95\% CI 1.22-3.36, $P=0.0069$; Table 2).

\section{Risk factors for delayed administration}

The univariate analysis to evaluate risk factors for the delayed administration of S-1 monotherapy after 8 weeks revealed that age $\geq 70$ years, upper third of the tumor location, the presence of postoperative complications, and hospital stay after surgery $\geq 16.9$ days were significant risk factors (Table 3). Moreover, the multivariate analysis identified the presence of postoperative complications as an independent risk factor for delayed S-1 monotherapy administration after 8 weeks (Odds ratio $3.75 ; 95 \%$ CI $1.74-8.09, P=0.0007$, Table 3). 
a

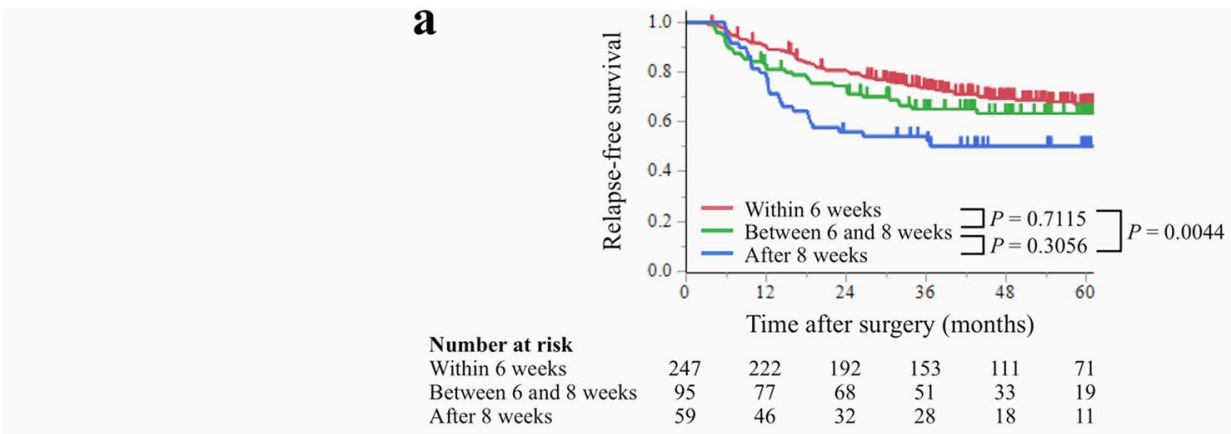

b

Stage II

Number at risk

Within 6 weeks

Between 6 and 8 weeks

After 8 weeks

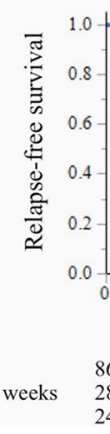

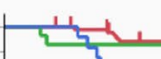

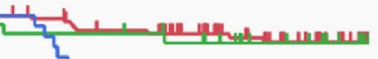

4

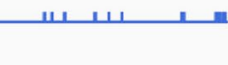

- Within 6 weeks

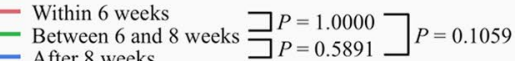

\begin{tabular}{ccccc} 
- After 8 weeks & \multicolumn{3}{c}{$\sqsupset P=0.5891$} \\
\hline 12 & 24 & 36 & 48 & 60
\end{tabular}

Time after surgery (months)

$\begin{array}{ccccc}83 & 76 & 65 & 48 & 30 \\ 26 & 26 & 23 & 13 & 7\end{array}$

$\begin{array}{lllll}26 & 26 & 23 & 13 & 7 \\ 23 & 18 & 16 & 12 & 7\end{array}$ c

Stage III

Number at risk

Within 6 weeks

Between 6 and 8 weeks

After 8 weeks

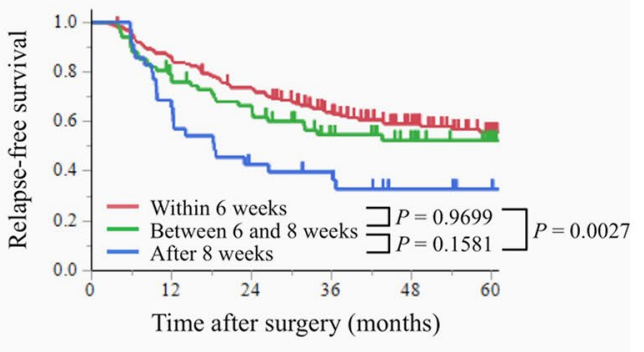

Time after surgery (months)

$\begin{array}{cccccc}161 & 138 & 116 & 88 & 63 & 41 \\ 67 & 51 & 42 & 28 & 20 & 12 \\ 35 & 23 & 14 & 12 & 6 & 4\end{array}$

Fig. 3 Relapse-free survival for each group. a All patients; b patients with stage II; c patients with stage III

\section{Recurrence site after surgery}

We investigated the patterns of the initial recurrence after surgery and found that the prevalence of overall recurrence was $30.0 \%$ in the administration within 6 weeks, $34.7 \%$ in the administration between 6 and 8 weeks, and $49.2 \%$ in the administration after 8 weeks groups, which were significantly different $(P=0.0196$, Fig. $4 a)$. The cumulative rate of hematogenous recurrence as an initial site was increased significantly compared with delayed administration: $7.3 \%$ in the administration within 6 weeks, $15.8 \%$ in the administration between 6 and 8 weeks, and $21.0 \%$ in the administration after 8 weeks groups (Fig. 4b: within 6 weeks vs. between 6 and 8 weeks, $P=0.0332$; within 6 weeks vs. after 8 weeks, $P=0.0003$; between 6 and 8 weeks vs. after 8 weeks, $P=0.905$ ).

\section{Subgroup analyses}

A forest plot to evaluate the impact of delayed administration after 8 weeks on recurrence is shown in Fig. 5. There was no interaction between treatment and any of these factors. Delayed administration after 8 weeks was a significant risk factor for recurrence in the following subgroups: male, larger tumor size ( $\geq 5.0 \mathrm{~cm}: n=214$, HR 1.955 ; 95\% CI 1.19-3.12, $P=0.0118)$, tumor differentiation, and pStage III $(n=263$, HR 2.03; 95\% CI 1.26-3.15, $P=0.0047$ ).

\section{Discussion}

In this study, we sought to clarify the prognostic impact of the timing for initiating postoperative chemotherapy using S-1 monotherapy in gastric cancer patients. S-1 monotherapy administration between 6 and 8 weeks has equivalent results compared with administration within 6 weeks for pathological stage II or III gastric cancer. However, administration after 8 weeks showed worse prognostic outcomes.

The benefit of postoperative adjuvant chemotherapy on prognosis is likely because of the eradication of residual micrometastasis deposits in a proportion of patients who would otherwise experience cancer recurrence [4-6]. The benefits achieved by adjuvant chemotherapy primarily occur during the period of drug administration, and although there is little additional benefit after drugs are withdrawn, the benefits continued for a short time [20]. Thus, it is necessary to eradicate residual microscopic cancer cells during drug administration, which requires a corresponding number of courses, and the duration of administration is important. This logic also applies to postoperative adjuvant chemotherapy for gastric cancer, and the importance of the duration of administration was also demonstrated in the Fujitani et al. study [7] and the OPAS-1 trial [8].

The proper timing for initiation of postoperative adjuvant chemotherapy with S-1 monotherapy in gastric cancer patients has not been fully evaluated. There were a few retrospective studies that suggested a correlation between the 
Table 2 Univariate and multivariable analyses for relapse-free survival

\begin{tabular}{|c|c|c|c|c|c|c|}
\hline \multirow[t]{2}{*}{ Variables } & \multicolumn{2}{|c|}{ Univariate } & \multirow[t]{2}{*}{$P$ value } & \multicolumn{2}{|c|}{ Multivariable } & \multirow[t]{2}{*}{$P$ value } \\
\hline & HR & $95 \%$ CI & & HR & $95 \%$ CI & \\
\hline Sex & & & 0.7911 & & & \\
\hline Male & 1 & & & & & \\
\hline Female & 1.05 & $0.72-1.50$ & & & & \\
\hline Age (years) & & & 0.0941 & & & \\
\hline$<70$ & 1 & & & & & \\
\hline$\geq 70$ & 1.34 & $0.95-1.89$ & & & & \\
\hline Tumor size $(\mathrm{cm})$ & & & $<0.0001$ & & & 0.0458 \\
\hline$<5.0$ & 1 & & & 1 & & \\
\hline$\geq 5.0$ & 2.02 & $1.42-2.90$ & & 1.45 & $1.01-2.10$ & \\
\hline Histological type & & & 0.1927 & & & \\
\hline Differentiated & 1 & & & & & \\
\hline Undifferentiated & 1.26 & $0.89-1.79$ & & & & \\
\hline Vessel infiltration & & & 0.0036 & & & 0.4159 \\
\hline Absent & 1 & & & 1 & & \\
\hline Present & 1.81 & $1.20-2.80$ & & 1.20 & $0.78-1.90$ & \\
\hline Lymphatic infiltration & & & 0.0030 & & & 0.1256 \\
\hline Absent & 1 & & & 1 & & \\
\hline Present & 3.08 & $1.40-8.70$ & & 1.95 & $0.84-5.68$ & \\
\hline Pathological stage & & & $<0.0001$ & & & $<0.0001$ \\
\hline II & 1 & & & 1 & & \\
\hline III & 4.56 & $2.82-7.86$ & & 3.85 & $2.33-6.77$ & \\
\hline Type of gastrectomy & & & 0.0024 & & & 0.1365 \\
\hline Non-total gastrectomy & 1 & & & 1 & & \\
\hline Total gastrectomy & 1.69 & $1.20-2.36$ & & 1.30 & $0.92-1.84$ & \\
\hline Performance status & & & 0.4095 & & & \\
\hline $0-1$ & 1 & & & & & \\
\hline $2-3$ & 1.49 & $1.53-3.28$ & & & & \\
\hline Postoperative complications ${ }^{\mathrm{a}}$ & & & 0.0398 & & & 0.9183 \\
\hline Absent & 1 & & & 1 & & \\
\hline Present & 1.49 & $1.02-2.14$ & & 1.02 & $0.66-1.57$ & \\
\hline \multicolumn{7}{|l|}{ Administration of S-1 } \\
\hline Within 6 weeks & 1 & & & 1 & & \\
\hline Between 6 and 8 weeks & 1.28 & $0.84-1.91$ & 0.2474 & 1.19 & $0.77-1.81$ & 0.4304 \\
\hline After 8 weeks & 1.96 & $1.26-2.98$ & 0.0036 & 2.05 & $1.22-3.36$ & 0.0069 \\
\hline
\end{tabular}

$H R$ hazard ratio, $C I$ confidence interval

${ }^{a}$ Grade 2 or greater using the Clavien-Dindo Classification timing for initiation of adjuvant chemotherapy and prognosis: early initiation (within 4 weeks after surgery) was not associated with a favorable prognosis [21], administration within 6 weeks after surgery was associated with a favorable prognosis [10], delayed initiation (more than 8 weeks after surgery) was associated with a worse prognosis [9], and the timing of initiation was not correlated with prognosis [7]. However, these reports cannot sufficiently eliminate the following confounding factors that affect prognosis: imbalance in the chemotherapy regimen $[9,21]$, lack of information about the duration of adjuvant chemotherapy [9, 21], and lack of information about postoperative complications [9].
The crucial problem is the duration of $\mathrm{S}-1$ administration, which is the major difference between our study and previous studies. The OPAS-1 trial [8] concluded that the benefit of S-1 monotherapy was insufficient in patients with a duration within 6 months. Based on this result, we excluded patients who had withdrawn from S-1 monotherapy within 6 months for reasons other than recurrence and investigated whether the timing of postoperative chemotherapy initiation affects gastric cancer patient prognosis. Thus, our results indicate that delayed S-1 monotherapy administration after 8 weeks was associated with a worse prognosis even if it was administered for a sufficient duration. 
Table 3 Potential risk factors for delayed administration of S-1 monotherapy after 8 weeks

\begin{tabular}{|c|c|c|c|c|c|c|c|}
\hline \multirow[t]{2}{*}{ Variables } & & \multicolumn{2}{|c|}{ Univariate } & \multirow[t]{2}{*}{$P$ value } & \multicolumn{2}{|c|}{ Multivariable } & \multirow[t]{2}{*}{$P$ value } \\
\hline & & OR & $95 \% \mathrm{CI}$ & & OR & $95 \% \mathrm{CI}$ & \\
\hline Sex & Male & 1.25 & $0.66-2.34$ & 0.4929 & & & \\
\hline Age (years) & $\geq 70$ & 1.76 & $1.01-3.07$ & 0.0471 & 1.74 & $0.96-3.16$ & 0.0698 \\
\hline Preoperative body mass index $\left(\mathrm{kg} / \mathrm{m}^{2}\right)$ & $\geq 22$ & 1.24 & $0.72-2.16$ & 0.4382 & & & \\
\hline Diabetes mellitus & Present & 1.61 & $0.81-3.19$ & 0.1708 & & & \\
\hline Cerebrovascular comorbidities & Present & 0.91 & $0.26-3.18$ & 0.8834 & & & \\
\hline Cardiopulmonary comorbidities & Present & 1.29 & $0.59-2.80$ & 0.5276 & & & \\
\hline Performance status & 0 & 0.80 & $0.38-1.69$ & 0.5574 & & & \\
\hline Type of gastrectomy & Total & 1.54 & $0.88-2.68$ & 0.1284 & & & \\
\hline Surgical approach & Laparoscopy & 0.96 & $0.32-2.88$ & 0.9472 & & & \\
\hline Lymph node dissection & D2 & 0.55 & $0.27-1.12$ & 0.0978 & & & \\
\hline Tumor location & Upper third & 1.92 & $1.06-3.49$ & 0.0322 & 1.54 & $0.80-2.96$ & 0.1929 \\
\hline Tumore size $(\mathrm{cm})$ & $\geq 5.0$ & 1.22 & $0.70-2.13$ & 0.4780 & & & \\
\hline Macroscopic type & Type 4 or 5 & 1.44 & $0.56-3.66$ & 0.4497 & & & \\
\hline Pathological stage & III & 0.73 & $0.41-1.28$ & 0.2741 & & & \\
\hline Intraoperative transfusion & Performed & 1.37 & $0.54-3.49$ & 0.5074 & & & \\
\hline Postoperative complications ${ }^{\mathrm{a}}$ & Present & 5.69 & $3.18-10.1$ & $<0.0001$ & 3.75 & $1.74-8.09$ & 0.0007 \\
\hline Hospital stay after surgery & $\geq 16.9$ days $^{\mathrm{b}}$ & 4.05 & $2.29-7.17$ & $<0.0001$ & 1.72 & $0.79-3.74$ & 0.1733 \\
\hline
\end{tabular}

OR Odds ratio, $C I$ confidence interval

${ }^{a}$ Grade II or greater using the Clavien-Dindo Classification

${ }^{\mathrm{b}}$ Mean days of the hospital stay after surgery in all patients
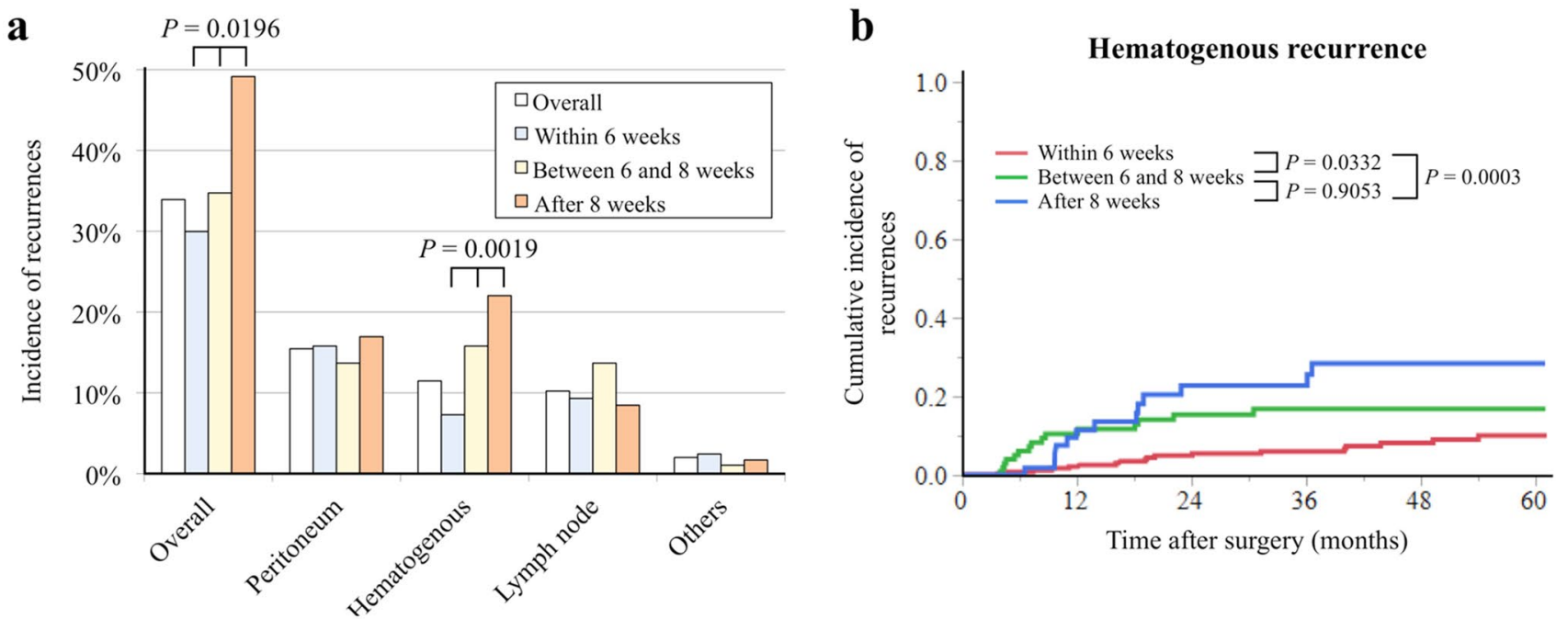

Fig. 4 a Frequencies of the sites of initial recurrence for each group. b Cumulative incidence of hematogenous recurrence as the initial site for each group

In our study, even in patients who received a sufficient duration of S-1 administration, the delayed administration of S-1 after 8 weeks was associated with a poor prognosis. This may be because the residual microscopic cancer cells increased exponentially during the delay in initiation of postoperative chemotherapy. Even if it was administered for a sufficient duration, the residual cancer cells could not be completely eradicated, which led to cancer recurrence. If there are more residual microscopic cancer cells after surgery, the recurrence rate should also be high. In our study, delayed administration after 8 weeks was a powerful poor prognostic factor and was related to recurrence during the period of S-1 administration or within 6 months after withdrawing S-1, indicating that it may be associated with more 


\section{Hazard ratio for relapse}

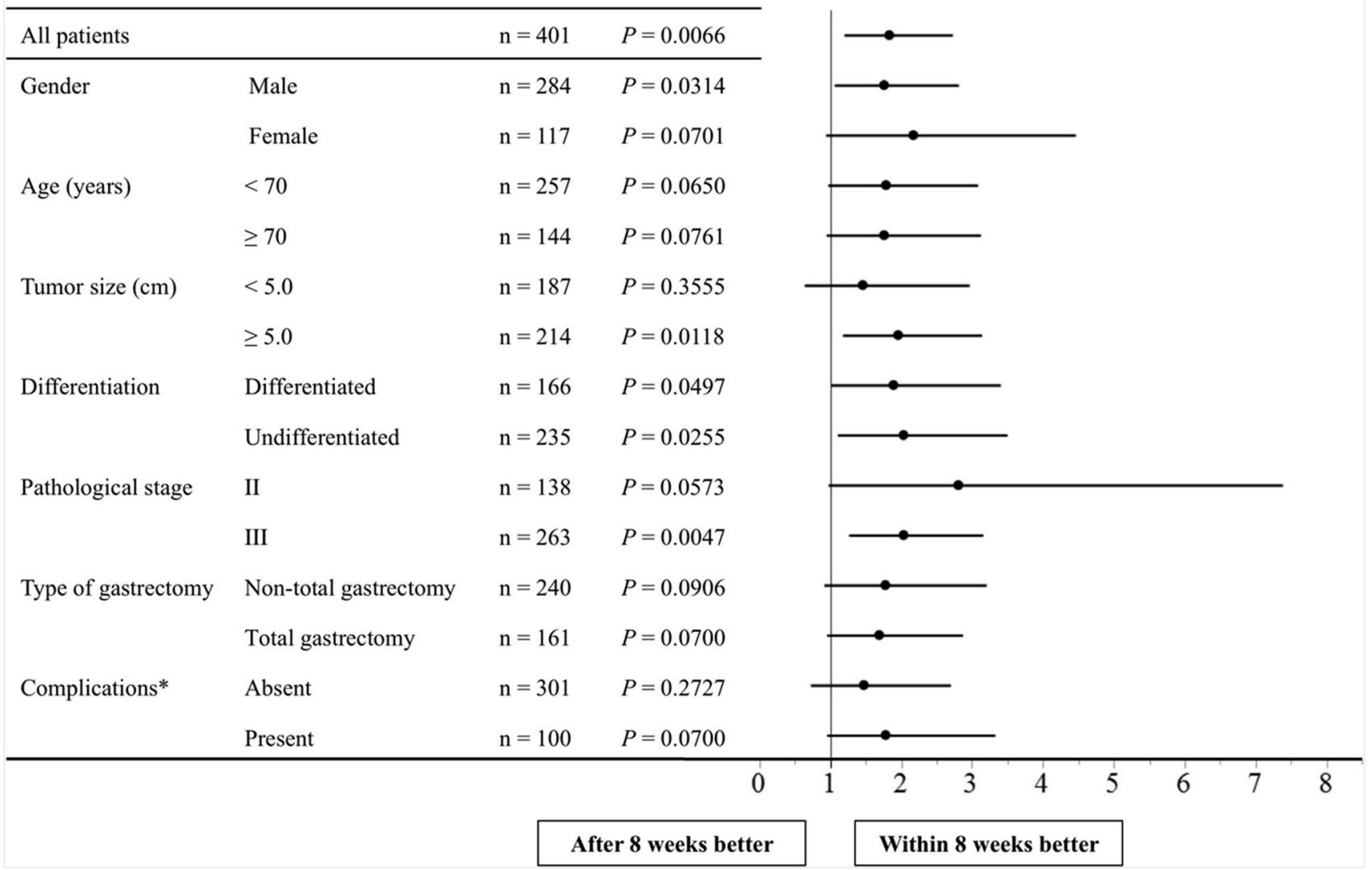

Fig. 5 Forest plot evaluating the impact of delayed S-1 administration after 8 weeks on relapse

residual microscopic cancer cells. An additional explanation could be that delayed administration after 8 weeks was associated with several factors that are related to hematogenous recurrence. Age, total gastrectomy, disease stage, and postoperative complications are reported to correlate with malnutrition and pro-inflammatory status, and poor status of these is related to hematogenous recurrence of gastric cancer [22]. In our study, most of these factors also caused a delay in the initiation of S-1 monotherapy. To eliminate these confounding factors, we performed a subgroup analysis and there was no interaction between the treatment and any of these factors, indicating that delayed administration after 8 weeks alone was also associated with poor prognosis, especially hematogenous recurrence.

Our study clarified that postoperative adjuvant chemotherapy using S-1 monotherapy should be started within 8 weeks. Additionally, we showed that the presence of postoperative complications was an independent risk factor for delayed administration after 8 weeks. However, we could not determine how to salvage patients who could not start postoperative chemotherapy within 8 weeks. It was reported that S-1 monotherapy mainly suppresses peritoneal recurrences, but its effects on hematogenous recurrence is weak [23]. Combination postoperative adjuvant chemotherapy for gastric cancer, such as capecitabine plus oxaliplatin, has been reported to significantly suppress hematogenous metastasis and it had a better effect than S-1 monotherapy in pStage III patients [24]. If the initiation of postoperative chemotherapy is delayed, it may be better to choose a strong chemotherapy regimen after waiting until the patient's general condition to improve and for chemotherapy to become available, or to choose postoperative S-1 monotherapy after more than 1 year. However, this question was not investigated, and it remains unanswered. In the future, a large-scale research study will be necessary to answer this new clinical question.

Our study has several limitations. First, our analysis is nonrandomized and retrospective in nature. Second, toxicity, dose reduction, compliance, and the relative dose intensity of S-1 were not evaluated. Third, the number of patients who received S-1 monotherapy after 8 weeks was limited to only 59 patients. Finally, patients who had recurrence while administration of S-1 could not begin were not included this study. However, we believe that our results are relevant 
because a randomized clinical trial that is designed to clarify this question would be unrealistic and unethical.

\section{Conclusion}

Our results indicate that delayed administration of S-1 monotherapy after 8 weeks was associated with a worse prognosis, even if S-1 administration was continued for more than 6 months. It was a strong prognostic factor in patients with pStage III and it was linked to increased prevalence of hematogenous metastasis as initial recurrent sites. Additionally, the presence of postoperative complications was an independent risk factor for delayed administration after 8 weeks. Therefore, surgeons and physicians should make efforts to avoid postoperative complications and continue S-1 monotherapy intermittently, and to begin this therapy within 8 weeks. Doing so will improve the patients' prognosis.

Acknowledgements We thank Edanz Group (www.edanzediting.com/ ac) for editing a draft of this manuscript.

\section{Compliance with ethical standards}

Conflict of interest The authors declare that they have no competing interests.

Ethical approval All procedures performed were in accordance with the ethical standards of the responsible committee on human experimentation (institutional and national) and with the Helsinki Declaration of 1964 and later versions.

Informed consent Informed consent was obtained from all individual participants included in the study.

\section{References}

1. Ferlay J, Soerjomataram I, Dikshit R, Eser S, Mathers C, Rebelo $\mathrm{M}$, et al. Cancer incidence and mortality worldwide: sources, methods and major patterns in GLOBOCAN 2012. Int J Cancer. 2015;136:E359-86.

2. Japanese Gastric Cancer A. Japanese gastric cancer treatment guidelines 2014 (ver 4). Gastric Cancer. 2017;20:1-19.

3. Sakuramoto S, Sasako M, Yamaguchi T, Kinoshita T, Fujii M, Nashimoto A, et al. Adjuvant chemotherapy for gastric cancer with S-1, an oral fluoropyrimidine. N Engl J Med. 2007;357:1810-20.

4. Okuyama T, Korenaga D, Edagawa A, Itoh S, Oki E, Kawanaka $\mathrm{H}$, et al. Prognostic effects of oral anti-cancer drugs as adjuvant chemotherapy for 2 years after gastric cancer surgery. Surg Today. 2012;42:734-40.

5. Aoyama T, Yoshikawa T. Adjuvant therapy for locally advanced gastric cancer. Surg Today. 2017;47:1295-302.

6. Sakamoto J, Morita S, Kodera Y, Rahman M, Nakao A. Adjuvant chemotherapy for gastric cancer in Japan: global and Japanese perspectives. Cancer Chemother Pharmacol. 2004;54(Suppl 1):S25-31.

7. Fujitani K, Kurokawa Y, Takeno A, Endoh S, Ohmori T, Fujita J, et al. Time to initiation or duration of S-1 adjuvant chemotherapy; which really impacts on survival in stage II and III gastric cancer? Gastric Cancer. 2018;21:446-52.
8. Yoshikawa T, Terashima M, Mizusawa J, Nunobe S, Nishida Y, Yamada T, et al. Four courses versus eight courses of adjuvant S-1 for patients with stage II gastric cancer (JCOG1104 [OPAS-1]): an open-label, phase 3, non-inferiority, randomised trial. Lancet Gastroenterol Hepatol. 2019;4:208-16.

9. Park HS, Jung M, Kim HS, Kim HI, An JY, Cheong JH, et al. Proper timing of adjuvant chemotherapy affects survival in patients with stage 2 and 3 gastric cancer. Ann Surg Oncol. 2015;22:224-31.

10. Yamamoto M, Sakaguchi Y, Kinjo N, Yamaguchi S, Egashira A, Minami K, et al. S-1 adjuvant chemotherapy earlier after surgery clinically correlates with prognostic factors for advanced gastric cancer. Ann Surg Oncol. 2016;23:546-51.

11. Tokunaga M, Tanizawa Y, Bando E, Kawamura T, Terashima M. Poor survival rate in patients with postoperative intra-abdominal infectious complications following curative gastrectomy for gastric cancer. Ann Surg Oncol. 2013;20:1575-83.

12. Aoyama T, Yoshikawa T, Hayashi T, Kuwabara H, Mikayama Y, Ogata T, et al. Risk factors for 6-month continuation of S-1 adjuvant chemotherapy for gastric cancer. Gastric Cancer. 2013;16:133-9.

13. Aoyama T, Yoshikawa T, Shirai J, Hayashi T, Yamada T, Tsuchida $\mathrm{K}$, et al. Body weight loss after surgery is an independent risk factor for continuation of S-1 adjuvant chemotherapy for gastric cancer. Ann Surg Oncol. 2013;20:2000-6.

14. Liu JY, Peng CW, Yang XJ, Huang CQ, Li Y. The prognosis role of AJCC/UICC 8(th) edition staging system in gastric cancer, a retrospective analysis. Am J Transl Res. 2018;10:292-303.

15. Dindo D, Demartines N, Clavien PA. Classification of surgical complications: a new proposal with evaluation in a cohort of 6336 patients and results of a survey. Ann Surg. 2004;240:205-13.

16. Clavien PA, Barkun J, de Oliveira ML, Vauthey JN, Dindo D, Schulick RD, et al. The Clavien-Dindo classification of surgical complications: five-year experience. Ann Surg. 2009;250:187-96.

17. Japanese Gastric Cancer A. Japanese classification of gastric carcinoma: 3rd English edition. Gastric Cancer. 2011;14:101-12.

18. Kanda M, Murotani K, Kobayashi D, Tanaka C, Yamada S, Fujii $\mathrm{T}$, et al. Postoperative adjuvant chemotherapy with $\mathrm{S}-1$ alters recurrence patterns and prognostic factors among patients with stage II/ III gastric cancer: a propensity score matching analysis. Surgery. 2015;158:1573-80.

19. Dienstmann R, Salazar R, Tabernero J. Personalizing colon cancer adjuvant therapy: selecting optimal treatments for individual patients. J Clin Oncol. 2015;33:1787-96.

20. Lewis JP. An interpretation of the EBCTCG data. Oncologist. 2007;12:505-9.

21. Lee Y, Min SH, Park KB, Park YS, Kim JW, Ahn SH, et al. Effect of early adjuvant chemotherapy on survival of advanced gastric cancer patients: a propensity score-matched analysis. J Gastric Cancer. 2018;18:58-68.

22. Sato B, Kanda M, Tanaka C, Kobayashi D, Iwata N, Hattori N, et al. Significance of preoperative systemic inflammation score in short-term and long-term outcomes of patients with pathological T2-4 gastric cancer after radical gastrectomy. World J Surg. 2018;42:3277-85.

23. Sasako M, Sakuramoto S, Katai H, Kinoshita T, Furukawa H, Yamaguchi T, et al. Five-year outcomes of a randomized phase III trial comparing adjuvant chemotherapy with $\mathrm{S}-1$ versus surgery alone in stage II or III gastric cancer. J Clin Oncol. 2011;29:4387-93.

24. Bang YJ, Kim YW, Yang HK, Chung HC, Park YK, Lee KH, et al. Adjuvant capecitabine and oxaliplatin for gastric cancer after D2 gastrectomy (CLASSIC): a phase 3 open-label, randomised controlled trial. Lancet (London, England). 2012;379:315-21.

Publisher's Note Springer Nature remains neutral with regard to jurisdictional claims in published maps and institutional affiliations. 